\title{
Kernel Anomalous Change Detection FOR REMOTE SENSING IMAGERY
}

\section{A PREPRINT}

\author{
José A. Padrón-Hidalgo \\ Image Processing Laboratory \\ Universitat de València \\ València, Spain \\ joseantoniopadronhidalgo@gmail.com
}

Nathan Longbotham

Descartes Lab

Santa Fe, NM, USA

nathan@descarteslabs.com

\author{
Valero Laparra \\ Image Processing Laboratory \\ Universitat de València \\ València, Spain \\ valero. laparra@uv.es
}

\author{
Gustau Camps-Valls \\ Image Processing Laboratory \\ Universitat de València \\ València, Spain \\ gcamps@uv.es
}

December 10, 2020

\begin{abstract}
Anomalous change detection is an important problem in remote sensing image processing. Detecting not only pervasive but anomalous or extreme changes has many applications for which methodologies are available. This paper introduces a nonlinear extension of a full family of anomalous change detectors based on covariance operators. In particular, this paper focuses on algorithms that utilize Gaussian and elliptically contoured distributions, and extend them to their nonlinear counterparts based on the theory of reproducing kernels in Hilbert spaces. The presented methods generalize their linear counterparts, based on the assumption of either Gaussian or elliptically-contoured distribution. We illustrate the performance of the introduced kernel methods in both pervasive and anomalous change detection problems involving both real and simulated changes in multi and hyperspectral imagery with different resolutions (AVIRIS, Sentinel-2, WorldView-2, Quickbird). A wide range of situations are studied, involving droughts, wildfires, and urbanization in real examples. Excellent performance in terms of detection accuracy compared to linear formulations is achieved, resulting in improved detection accuracy and reduced false alarm rates. Results also reveal that the ellipticallycontoured assumption may be still valid in Hilbert spaces. We provide an implementation of the algorithms as well as a database of natural anomalous changes in real scenarios.
\end{abstract}

\section{Introduction}

The problem of change detection deals with identifying transitions between a pair (or a series) of co-registered images $[42,52]$. Change detection in remote sensing images is of paramount relevance because it automatizes traditionally manual tasks in disaster management (floods, droughts and wildfires), helps in designing development and settlement plans as well as in urban and crop monitoring. Multitemporal classification and change detection are very active fields nowadays because of the increasing availability of complete time series of images and the interest in monitoring changes occurring on the Earth's cover due to either natural or anthropogenic activities. Complete constellations of civil and military satellites sensors currently provide with high spatial resolution and revisiting frequency.

\footnotetext{
${ }^{1} \hat{A} \odot$ IEEE. Personal use of this material is permitted. Permission from IEEE must be obtained for all other users,including reprinting/republishing this material for advertising or promotional purposes, creating new collective works for resale or redistribution to servers or lists, or reuse of any copyrighted components of this work in other works. DOI: 10.1109/TGRS.2019.2916212.
} 
The Copernicus' Sentinels ${ }^{2}$ or NASA's A-train ${ }^{3}$ programs are producing near real-time coverage of the globe. NASA is currently producing a Harmonized Landsat Sentinel-2 (HLS) data set, which can be used for monitoring agricultural resources with an unprecedented combination of $30 \mathrm{~m}$ spatial resolution and 2-3 days revisit. In parallel, new commercial satellite missions are being deployed to provide multispectral data at both high spatial and high temporal resolution. For example, the PlanetScope constellation by Planet Labs, Inc. can provide $5 \mathrm{~m}$ data daily for sites requested by the client, and the recently announced UrtheDaily constellation, specifically designed for operational agricultural applications, will acquire S2-like data also at 5-m spatial resolution and with an impressive full global coverage every day. It goes without saying that closed-range applications using drones and all kind of unmanned automated vehicles (UAVs) also challenge the field of automatic change detection. All in all, automatic image analysis in general and change detection in particular are becoming strictly necessary in the current era of data deluge.

The field of change detection (CD) methods is vast and many approaches are available in the literature $[28,32,33,43]$. A simple taxonomy could organize them according to three types of products [17, 52]: 1) binary maps, 2) detection of types of changes, and 3) full multiclass change maps, thus including classes of changes and unchanged land-cover classes. Each type of product can be achieved using different sources of information retrieved from the initial spectral images at time instants $t_{1}$ and $t_{2}$. Unsupervised CD has been widely studied, mainly because it meets the requirements of most applications: i) the speed in retrieving the change map and ii) the absence of labeled information in general applications. However, the lack of labeled information makes the problem of detection more difficult and thus unsupervised methods typically consider binary change detection problems only. In the last decade, change vector analysis (CVA) techniques have been widely applied: CVA converts the difference image to polar coordinates and operate in such representation space [6,31]. In [18], morphological operators were successfully applied to increase the discriminative power of the CVA method. In [5], a contextual parcel-based multiscale approach to unsupervised CD was presented. Traditional CVA relies on the experience of the researcher for the threshold definition, and is still on-going research $[15,23]$. The method has been also studied in terms of sensitivity to differences in registration and other radiometric factors [7]. Another interesting approach based on spectral transforms is the multivariate alteration detection (MAD) [35,37], where canonical correlation is computed for the points at each time instant and then subtracted. The method consequently reveals changes invariant to linear transformations between the time instants. Radiometric normalization issues for MAD has been recently considered in [11], and nonlinear extensions have been also realized via kernel machines (KM) [21,38]. Other approaches based on KM have proposed to use dimensionality reduction via principal components [19] or slow features [61] of the difference image. Clustering has been used in recent binary CD. In [13], rather than converting the difference image in the polar domain, local PCAs are used in sub-blocks of the image, followed by a binary $k$-means clustering to detect changed/unchanged areas locally. Kernel clustering has been also studied in $[59,60]$, where kernel $k$-means with parameters optimized using an unsupervised ANOVA-like cost function is used to separate the two clusters in a fully unsupervised way. Finally, unsupervised neural networks have been considered for binary CD [40,41]. In [20], a Hopfield neural network, where each neuron is connected to a single pixel is used to enforce neighborhood relationships. Lately, many efforts have been conducted in using deep convolutional neural networks as well [27,39,62].

When the signature of the class to be detected is unknown, anomaly detection (AD) algorithms are more often used as an alternative to traditional $\mathrm{CD}$. $\mathrm{AD}$ reduces to assuming (and/or defining) a model for the background class, and then looking for the test samples lying far from the mean of the background distribution. Depending on the assumptions made on the background, different anomaly detectors can be used. If the background is modeled as a Gaussian distribution, the Reed-Xiaoli (RX) $[29,44]$ detector can be used. The RX detector identifies outliers by computing the Mahalanobis distance between the considered pixel and the background mean vector. If the background is believed to be more complex, for instance multi-Gaussian, it can be modeled with a Gaussian mixture model [3]. Application of these principles to the detection of small objects in series of remote sensing images can be found in [12]. The RX detector has been extended to its nonlinear kernelized version in [25], and many variants have been introduced in the last years [63].

A related field of investigation in this direction is the so-called anomalous change detection (ACD) [53]: in this field, one looks for changes that are interestingly anomalous in multitemporal series of images, and try to highlight them in contrast to acquisition condition changes, registration noise, or seasonal variation. The interest in ACD is high, and many methods have been proposed in the literature, ranging from regression-based approaches like in the chronocrome [47] where big ('influential') residuals are associated with anomalies [4,16], to equalization-based approaches that rely on whitening principles [34], as well as multivariate methods [2] that reinforce directions in feature spaces associated with noisy or rare events $[22,36]$. The work [56] formalized the field by introducing a framework for ACD, which assumes Gaussianity, yet the derived detector delineates hyperbolic decision functions. Even though the Gaussian assumption reports some advantages (e.g. tractability and generally good performance) it

\footnotetext{
${ }^{2}$ http://www.esa.int/esaLP/SEMZHM0DU8E_LPgmes_0.html

${ }^{3}$ http://www.nasa.gov/mission_pages/a-train/a-train.html
} 
is still an ad hoc assumption that it is not necessarily fulfilled in practice. This is the motivation in [57], where the authors introduced elliptically-contoured (EC) distributions that generalize the Gaussian distribution and proved more appropriate to modeling fatter tail distributions and thus detect anomalies more effectively. The EC decision functions are point-wise nonlinear and still rely on second-order feature relations. Recent advances in ACD have considered methods robust to pixel misregistration [58] and sequences of several images in RX and chronocrome settings [55].

In this paper, we extend the family of ACD methods in [57] to cope with higher-order feature relations through the theory of reproducing kernels. Kernel methods allow the generalization of algorithms that are expressed in terms of dot products to account for higher-order (nonlinear) feature relations, yet still relying on linear algebra [10,46,50]. We illustrate the performance of the introduced kernel ACD methods in different experiments involving synthetic, artificially enforced and natural anomalous changes in multi- and hyperspectral imagery with different spatial resolutions (AVIRIS, Sentinel-2, WorldView-2, Quickbird). A wide range of situations are studied, involving droughts, wildfires, and urbanization in real examples. Very good performance is achieved in terms of detection accuracy compared to the linear formulations. Results also reveal that the elliptically-contoured assumption may be still valid in Hilbert spaces, even when high pervasive distortions mask anomalous targets.

The rest of the paper is outlined as follows. Section 2 reviews the family of (Gaussian and elliptically-contoured) ACD algorithms introduced in [57], and introduces the proposed nonlinear (kernel-based) versions. The introduced methods generalize the previous ones for the linear kernel, and provide more flexible mappings to account for higherorder dependencies between features. Section 3 presents experiments comparing the performance of the proposed algorithms with their linear counterpart in different scenarios. Finally, section 4 concludes the paper.

\section{Proposed Family of Kernel Anomaly Change Detection Methods}

An anomaly can be loosely defined as a sample with small probability to occur. An anomalous change is thus a rare, unexpected, change between two consecutive observations of the same phenomenon. In this paper we want to find samples that can be interpreted as anomalous changes between two multidimensional images. This calls for studying and characterizing differences between multivariate distributions, and in particular those features that account for the anomalous changes. In [57] a framework to define different anomalous change detectors based on probability distributions was formalized.

Let treat the two images as random variables $X$ and $Y$, with probability distributions $\mathbb{P}_{X}$ and $\mathbb{P}_{Y}$ respectively. Let us indicate the joint distribution as $\mathbb{P}_{X, Y}$ which accounts for how probable particular joint events are. The marginal distributions $\mathbb{P}_{X}$ and $\mathbb{P}_{Y}$ can be used in order to mitigate the problem of detecting a joint anomaly as an anomalous change. Given two pixels $\mathbf{x}_{i}, \mathbf{y}_{i} \in \mathbb{R}^{d}$ from the same spatial location $i$ but each one from one image, the general formula to compute the amount of anomalousness of a change is:

$$
\mathcal{A}^{*}{ }_{X, Y}\left(\mathbf{x}_{i}, \mathbf{y}_{i}\right)=\frac{\mathbb{P}_{X}\left(\mathbf{x}_{i}\right) \mathbb{P}_{Y}\left(\mathbf{y}_{i}\right)}{\mathbb{P}_{X, Y}\left(\mathbf{x}_{i}, \mathbf{y}_{i}\right)} .
$$

A sample is detected as anomalous change when it is anomalous with respect to the joint distribution but not anomalous with respect to the distributions of each isolated image. We are using here all three distributions, however different combinations can be used as we will see below.

Usually equation 1 is applied by taking logarithms, $\mathcal{A}_{[X, Y]}\left(\mathbf{x}_{i}, \mathbf{y}_{i}\right)=\log \left(\mathcal{A}^{*}{ }_{[X, Y]}\left(\mathbf{x}_{i}, \mathbf{y}_{i}\right)\right)$, which can be interpreted in information theoretic terms by noting the relation between probability and information. Elaborating on Shannon's information [49] we have:

$$
\mathcal{A}_{X, Y}\left(\mathbf{x}_{i}, \mathbf{y}_{i}\right)=I_{X, Y}\left(\mathbf{x}_{i}, \mathbf{y}_{i}\right)-I_{X}\left(\mathbf{x}_{i}\right)-I_{Y}\left(\mathbf{y}_{i}\right),
$$

where $I_{A}\left(B_{i}\right)$ is the amount of information in Shannon's terms the sample $B_{i}$ provides assuming it follows the distribution $\mathbb{P}_{A}$. A sample could be interpreted as an anomalous change if the information obtained by observing both images simultaneously gives high information with respect to the information given by observing each isolated image.

\subsection{Linear ACD algorithms}

Assuming that all three distributions follow a multivariate Gaussian we can express the formula only in terms of covariance matrices. The amount of anomalousness is given by:

$$
\mathcal{A}_{\mathcal{G}}\left(\mathbf{x}_{i}, \mathbf{y}_{i}\right)=\xi\left(\mathbf{z}_{i}\right)-\beta_{x} \xi\left(\mathbf{x}_{i}\right)-\beta_{y} \xi\left(\mathbf{y}_{i}\right),
$$

where $\xi(\mathbf{a})=\mathbf{a}^{\top} \mathbf{C}_{a}^{-1} \mathbf{a}, \mathbf{C}_{a}$ is the estimated covariance matrix with the available data, and being $\mathbf{z}=[\mathbf{x}, \mathbf{y}] \in \mathbb{R}^{2 d}$. The value of $\beta_{x}, \beta_{y} \in\{0,1\}$ parameters defines which distributions are taken into account to define our anomaly. The different combinations give rise to different anomaly detectors (see Table 1). These methods and some variants 
Table 1: A family of ACD algorithms.

\begin{tabular}{||l|c|c||}
\hline \hline ACD algorithm & $\beta_{x}$ & $\beta_{y}$ \\
\hline \hline RX & 0 & 0 \\
Chronocrome $\mathbf{y} \mid \mathbf{x}$ & 0 & 1 \\
Chronocrome $\mathbf{x} \mid \mathbf{y}$ & 1 & 0 \\
Hyperbolic ACD & 1 & 1 \\
\hline
\end{tabular}
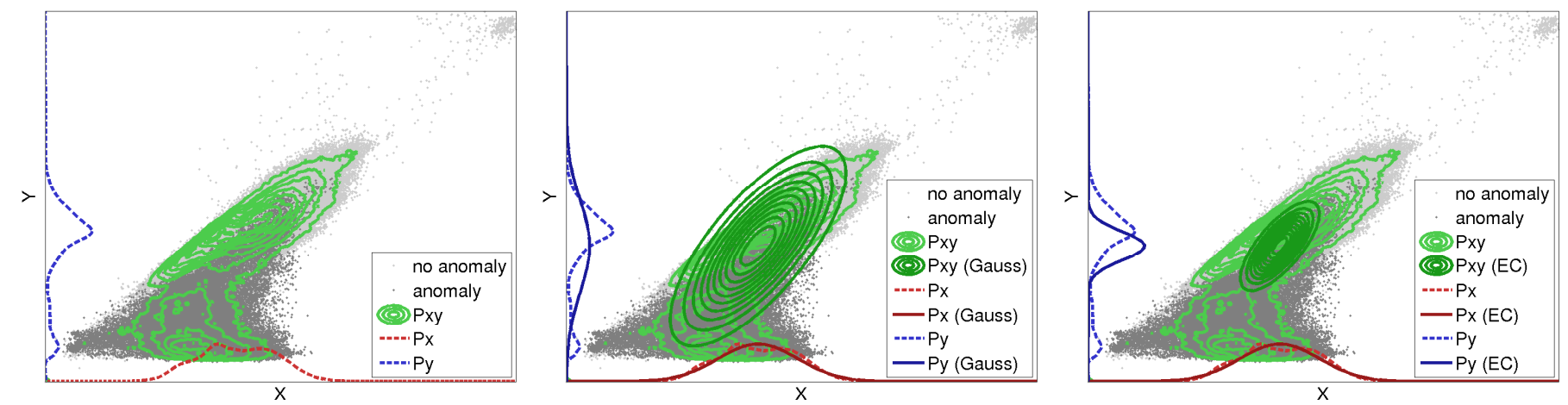

Figure 1: Illustration of ACD probabilistic framework. From left to right joint and marginal probability distributions of: the original data, Gaussian model, and Elliptically Contoured model. See text for details on the data.

have been widely used in many hyperspectral image analysis settings because of its simplicity and generally good performance $[14,24,45]$.

However, these methods are hampered by a fundamental problem: the (typically strong) assumption of Gaussianity that is implicit in the formulation. Accommodating other data distributions may not be easy in general. Theiler et al. [57] introduced alternative ACD to cope with elliptically-contoured distributions [8]: roughly speaking, the idea is to model the data using an elliptically-contoured (EC) distribution. EC distributions are particularly convenient in the case of images [30]. In particular the formulation introduced in [57] uses the multivariate Student's t-distribution, giving rise to the following formula for computing the amount of EC anomalousness:

$$
\begin{aligned}
\mathcal{A}_{\mathrm{EC}}\left(\mathbf{x}_{i}, \mathbf{y}_{i}\right) & =(2 d+\nu) \log \left(1+\frac{\xi\left(\mathbf{z}_{i}\right)}{\nu}\right) \\
& -\beta_{x}(d+\nu) \log \left(1+\frac{\xi\left(\mathbf{x}_{i}\right)}{\nu}\right) \\
& -\beta_{y}(d+\nu) \log \left(1+\frac{\xi\left(\mathbf{y}_{i}\right)}{\nu}\right),
\end{aligned}
$$

where $\nu$ controls the shape of the Student's t-distribution: for $\nu \rightarrow \infty$ the solution approximates the Gaussian and for $\nu \rightarrow 0$ it diverges.

An interesting particular case is the RX algorithm which brings to the same result for the Gaussian and the elliptical case (independently of the $\nu$ value). All extra operations applied by the EC formulation with regard to the Gaussian version are increasing monotonic functions which do not change the ordering of the values. Therefore, although the value of anomalousness are different (i.e. $\left.\mathcal{A}_{\mathcal{G}}\left(\mathbf{x}_{i}, \mathbf{y}_{i}\right) \neq \mathcal{A}_{\mathrm{EC}}\left(\mathbf{x}_{i}, \mathbf{y}_{i}\right)\right)$, the values are sorted in the same way which makes the detection curves also equal. The same effect happen between the RX methods based on kernels proposed in the next section.

Figure 1 shows an example of the distributions involved in the anomalous change detection framework. In order to be able to visualize the distributions we restrict ourselves to the most simple situation where each image contains just one band. In particular we show the distributions for the band 9 of a Sentinel-2 image over Australia, see table 4. We show results for the distribution of the data estimated using histograms, and when assuming Gaussian or EC distributions. Note that the estimation of the distribution based on histograms is only feasible in the low dimensional (i.e. 2D) case: when the number of bands increases the computation of the histogram becomes unfeasible due to the curse of dimensionality. However, the Gaussian and the EC model can be estimated easily for multiple dimensions. The difference between the Gaussian and the EC model relies in the kurtosis of the distribution, while in the Gaussian case 
is fixed in the EC case can be controlled with the $\nu$ parameter. By comparing the marginal distributions in the central and the right panels we can easily spot the differences between the Gaussian and the EC model. For the horizontal axes the data follows quite well the Gaussian model, i.e. the red solid line and the dashed red line are very similar in the central panel. However the Gaussian model fails when reproducing the probability for the vertical axes (central panel blue lines). Although it is not a perfect model, the EC distribution allows to get better description of the vertical axes (right panel blue lines), and simultaneously obtains a similar (good) result as the Gaussian model in the horizontal axes right panel red lines).

\subsection{Kernel ACD algorithms}

Previous methods are linear and depend on estimating covariance matrices with the available data, and use them as a metric for testing anomalousness. These methods are fast to apply, delineate point-wise nonlinear decision boundaries, but still rely on second-order statistics. This restricts the class of functions that can be implemented and thus the generalization capabilities of the algorithm. For instance in Fig. 1 the assumed joint distributions (dark green) for both Gaussian and EC models clearly differ from the real distribution (light green). We here address this issue through the theory of reproducing kernel functions [50], which allow us to capture higher-order feature relations while still relying on linear algebra. Kernel methods are particularly robust to reduced sample sizes and high-dimensional feature spaces, situations often encountered in hyperspectral image detection problems.

As the covariance matrix, Kernel methods rely on the notion of similarity between points in a higher (possibly infinite) dimensional space. They assume the existence of a Hilbert space $\mathcal{H}$ equipped with an inner product $\langle\cdot, \cdot\rangle_{\mathcal{H}}$. Samples in $\mathcal{X}$ are mapped into $\mathcal{H}$ by means of a feature map $\phi: \mathcal{X} \rightarrow \mathcal{H}, \mathbf{x}_{i} \mapsto \phi\left(\mathbf{x}_{i}\right), 1 \leq i \leq n$. The mapping function $\phi$ can be defined explicitly or implicitly, which is usually the case in kernel methods. The similarity between the elements in $\mathcal{H}$ can be estimated using its associated inner product $\langle\cdot, \cdot\rangle_{\mathcal{H}}$ via reproducing kernels in Hilbert spaces, $k: \mathcal{X} \times \mathcal{X} \rightarrow \mathbb{R}$, such that pairs of points $\left(\mathbf{x}_{i}, \mathbf{x}_{j}\right) \mapsto k\left(\mathbf{x}_{i}, \mathbf{x}_{j}\right)$. So we can estimate similarities in $\mathcal{H}$ without the explicit definition of the feature map $\phi$, and hence without the need of having access to the points in $\mathcal{H}$. The kernel function $k$ requires to satisfy Mercer's Theorem [1].

The mapped training data matrix $\mathbf{X}=\left[\mathbf{x}_{1}, \ldots \mathbf{x}_{n}\right] \in \mathbb{R}^{n \times d}$ is now denoted as $\boldsymbol{\Phi}=\left[\boldsymbol{\phi}\left(\mathbf{x}_{1}\right), \ldots \phi\left(\mathbf{x}_{n}\right)\right] \in \mathbb{R}^{n \times d_{\mathcal{H}}}$. In the following we show how to estimate the $\xi\left(\mathbf{x}_{i}\right)$ function in the Hilbert space, i.e. $\xi^{\mathcal{H}}\left(\mathbf{x}_{i}\right)=\xi\left(\phi\left(\mathbf{x}_{i}\right)\right)$. The other terms are derived equivalently. Note that one could think of different mappings for each image, $\phi: \mathbf{x} \rightarrow \phi(\mathbf{x})$ and $\boldsymbol{\psi}: \mathbf{y} \rightarrow \boldsymbol{\psi}(\mathbf{y}), \Psi \in \mathbb{R}^{n \times d_{\mathcal{F}}}$, respectively. However, in our case, we are forced to consider mapping to the same Hilbert space because we have to stack the mapped vectors to estimate $\xi(\phi(\mathbf{z}))$, i.e. $\mathcal{F}=\mathcal{H}$. The mapped training data to Hilbert spaces are denoted as $\boldsymbol{\Phi}$. In order to estimate $\xi\left(\phi\left(\mathbf{x}_{i}\right)\right)$ we follow the same procedure as in the linear case but first mapping the points to the Hilbert space

$$
\xi^{\mathcal{H}}\left(\mathbf{x}_{i}\right)=\phi\left(\mathbf{x}_{i}\right)\left(\boldsymbol{\Phi}^{\top} \boldsymbol{\Phi}\right)^{-1} \phi\left(\mathbf{x}_{i}\right)^{\top}
$$

Note that we do not have access to either the samples or the covariance in the Hilbert. However note that $\left(\boldsymbol{\Phi}^{\top} \boldsymbol{\Phi}\right)^{-1}=$ $\boldsymbol{\Phi}^{\top}\left(\boldsymbol{\Phi} \boldsymbol{\Phi}^{\top} \boldsymbol{\Phi} \boldsymbol{\Phi}^{\top}\right)^{-1} \boldsymbol{\Phi}$. This can be easily shown by right multipliying by the term $\boldsymbol{\Phi}^{\top} \boldsymbol{\Phi} \boldsymbol{\Phi}^{\top}$ and applying some linear algebra. By substituting in eq. 4 we get

$$
\xi^{\mathcal{H}}\left(\mathbf{x}_{i}\right)=\phi\left(\mathbf{x}_{i}\right) \boldsymbol{\Phi}^{\top}\left(\boldsymbol{\Phi} \boldsymbol{\Phi}^{\top} \boldsymbol{\Phi} \boldsymbol{\Phi}^{\top}\right)^{-1} \boldsymbol{\Phi} \phi\left(\mathbf{x}_{i}\right)^{\top}
$$

In this equation we can replace all dot products by reproducing kernel functions using the representer theorem [50], and hence

$$
\xi^{\mathcal{H}}\left(\mathbf{x}_{i}\right)=\xi\left(\phi\left(\mathbf{x}_{i}\right)\right)=\mathbf{k}_{i}(\mathbf{K K})^{-1} \mathbf{k}_{i}^{\top}
$$

where $\mathbf{k}_{i} \in \mathbb{R}^{1 \times n}$ contains the similarities between $\mathbf{x}_{i}$ and all training data, $\mathbf{X}$, and $\mathbf{K} \in \mathbb{R}^{n \times n}$ stands for the kernel matrix containing all training data similarities. The solution may need extra regularization $\xi^{\mathcal{H}}\left(\mathbf{x}_{i}\right)=\mathbf{k}_{i}(\mathbf{K K}+$ $\left.\lambda \mathbf{I}_{n}\right)^{-1} \mathbf{k}_{i}^{\top}, \lambda \in \mathbb{R}^{+}$. Therefore the kernel version of Eq. (2) is:

$$
\mathcal{A}_{\mathcal{G}}^{\mathcal{H}}\left(\mathbf{x}_{i}, \mathbf{y}_{i}\right)=\xi^{\mathcal{H}}\left(\mathbf{z}_{i}\right)-\beta_{x} \xi^{\mathcal{H}}\left(\mathbf{x}_{i}\right)-\beta_{y} \xi^{\mathcal{H}}\left(\mathbf{y}_{i}\right)
$$

By following a similar procedure for Eq. (3), one obtains kernel versions of the elliptically-contoured linear solution:

$$
\begin{aligned}
\mathcal{A}_{\mathrm{EC}}^{\mathcal{H}}\left(\mathbf{x}_{i}, \mathbf{y}_{i}\right) & =(2 d+\nu) \log \left(1+\frac{\xi^{\mathcal{H}}\left(\mathbf{z}_{i}\right)}{\nu}\right) \\
& -\beta_{x}(d+\nu) \log \left(1+\frac{\xi^{\mathcal{H}}\left(\mathbf{x}_{i}\right)}{\nu}\right) \\
& -\beta_{y}(d+\nu) \log \left(1+\frac{\xi^{\mathcal{H}}\left(\mathbf{y}_{i}\right)}{\nu}\right)
\end{aligned}
$$


RX (0.92)

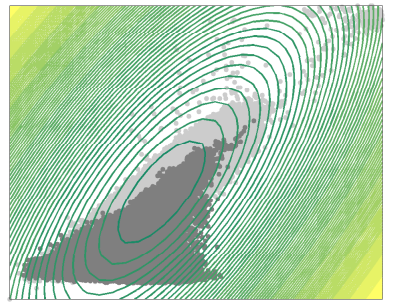

EC-RX (0.92)

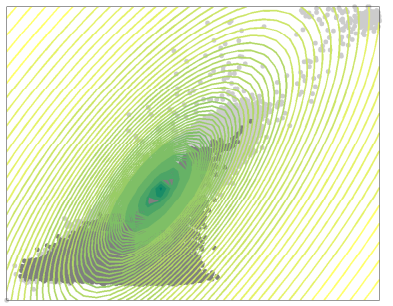

K-RX (0.95)

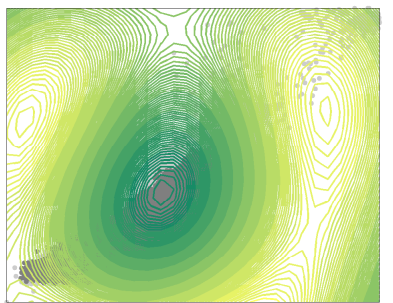

K-EC-RX (0.95)

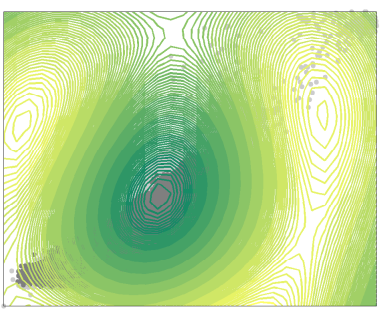

XY (0.54)

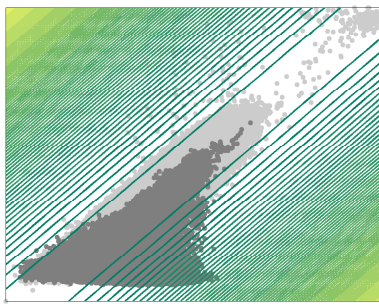

EC-XY (0.68)

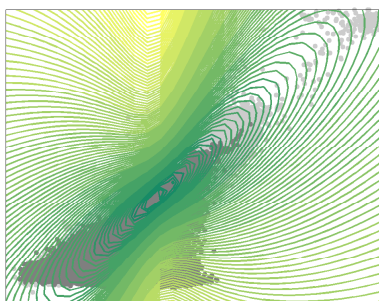

K-XY (0.89)

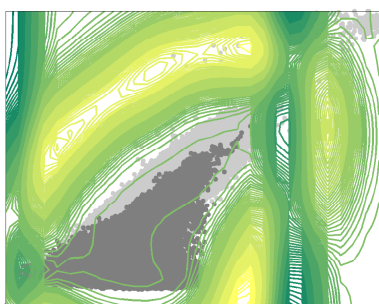

K-EC-XY (0.90)

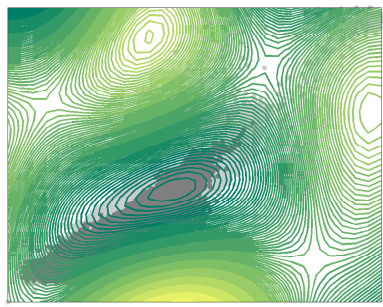

YX (0.81)

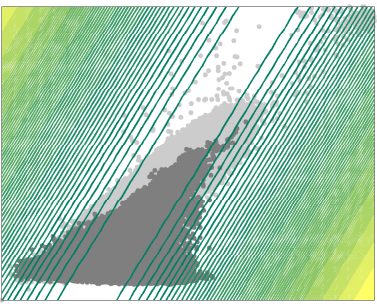

EC-YX (0.88)

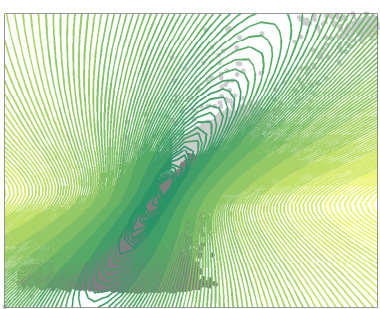

K-YX (0.92)

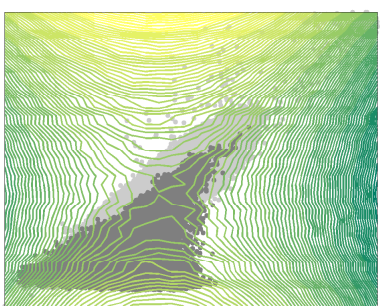

K-EC-YX (0.97)

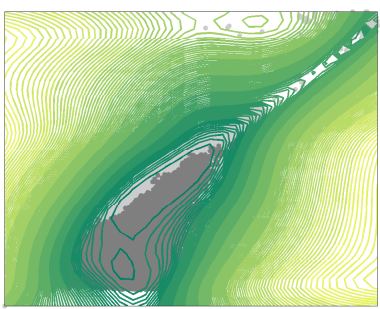

HACD (0.33)

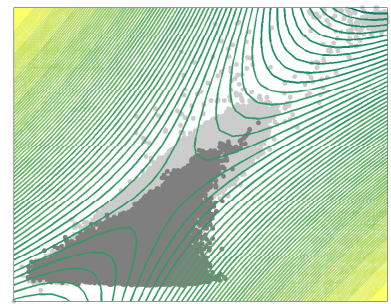

EC-HACD (0.43)

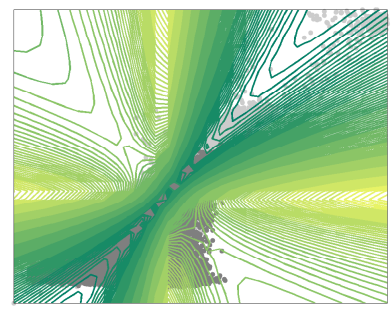

EC-HACD (0.95)

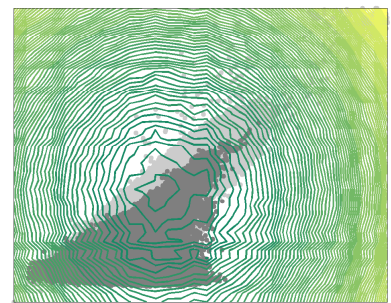

K-EC-HACD (0.96)

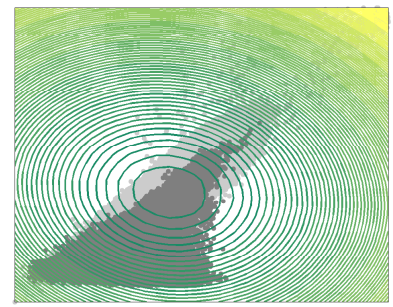

Figure 2: Toy example of anomalous detection surfaces for different methods, only the band 9 of a Sentinel-2 image was employed. Yellowish values mean more anomalous sample (i.e. bigger $\mathcal{A}$ ). Bright grey dots represent the non-anomalous data, while the dark gray are the anomalous data. Overall area under curve (AUC) of the receiver operating characteristic (ROC) values are given in parenthesis.

Note that in the case of $\beta_{x}=\beta_{y}=0$, the algorithm reduces to kernel RX which was previously introduced in [26].

Figure 2 shows an illustrative example of the surface, $\mathcal{A}$, for different methods. As in Fig. 1 the experiment is done using one band in order to be able of represent the surfaces. Different methods obtain different results, as we will see in the experiments the kernel methods obtain better results than their linear counterpart. The surfaces are direct consequence of the probabilistic model assumed, for instance in the case of RX for Gaussian and EC assumptions the surfaces are equivalent to the probabilistic distributions of $\mathbb{P}_{X, Y}$ in Fig. 1. It is clear that the kernel versions have much more capacity to non-linearly adapt the decision surface to the problem.

\section{Experimental Results}

This section analyzes the proposed methods in several simulated and real examples of pervasive and anomalous changes. We evaluate the performance of the methods by using the area under the curve (AUC) of the detection receiver operating characteristic (ROC) curves. 

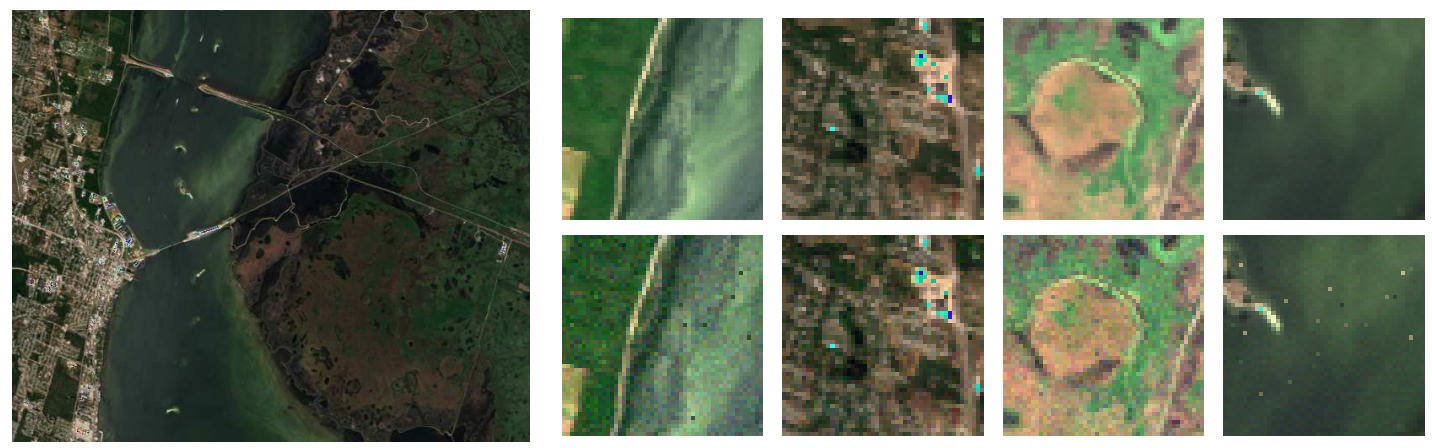

Figure 3: AVIRIS hyperspectral image (left panel), and four illustrative chips of simulated changes (right panel). The original (leftmost) image is used to simulate an anomalous change image (rightmost) by adding Gaussian noise and randomly scrambling $1 \%$ of the pixels.

We perform three experiments with datasets with different complexity and control on the analyzed changes. First, we perform an experiment where we control the anomalous change in a synthetic controlled scenario. The second experiment deals with data where the changes were real but manually introduced directly in the images. Finally, in the third battery of experiments we deal with natural changes related to floods, droughts and man-made changes.

We provide Matlab implementations of the methods. Moreover we made available a database with the labeled images employed in the third experiment ${ }^{4}$.

\subsection{Experiment 1: Simulated Changes}

This experiment is devoted to analyzing the capacity of the methods to detect pervasive and anomalous changes in simulated data by reproducing the simulation framework used in [54]. The data set (see Fig. 3) is an AVIRIS 224channel image acquired over the Kennedy Space Center (KSC), Florida, on March 23rd, 1996. The data was acquired from an altitude of $20 \mathrm{~km}$ and has a spatial resolution of $18 \mathrm{~m}$. After removing low SNR and water absorption bands, a total of 176 bands remain for analysis. More information can be found at http://www.csr.utexas.edu/. While the linear methods suffer from the dimensionality of the data and dimensionality reduction based on Principal Components Analysis (PCA) is usually performed as preprocessing [54], kernel methods are less sensitive to the dimensionality. While this type of preprocessing is usually accepted, it also involves the eliminatation of information. Here we did not further reduce the dimensionality with PCA and, instead, work directly with the SNR filtered hyperspectral data. Pervasive changes are simulated by adding Gaussian noise with 0 mean and 0.1 standard deviation to all the bands and all the pixels. The image with the added noise is taken as the second image. Anomalous changes are produced by scrambling some pixels in the second image. Note that since we are only switching the position of pixels the global distribution of the image does not change. Since the methods are applied pixel-wise, this yields anomalous changes that can not be detected as anomalies in the individual images.

In this experiment, we restrict ourselves to the use of hyperbolic detectors (HACD), i.e. $\beta_{x}=\beta_{y}=1$, that have shown improved performance for this particular experiment [57]. We tuned all the involved parameters (estimated covariance $\mathbf{C}_{z}$ and kernel $\mathbf{K}_{z}, \nu$ for the EC methods, lengthscale $\sigma$ parameter for the kernel versions) through standard crossvalidation in the training set and show results on the independent test set.

In this experiment we use the spectral angle mapper (SAM) kernel, $k\left(\mathbf{x}_{i}, \mathbf{x}_{j}\right)=$ $\exp \left(-\operatorname{acos}\left(\mathbf{x}_{i}^{\top} \mathbf{x}_{j} /\left(\left\|\mathbf{x}_{i}\right\|\left\|\mathbf{x}_{j}\right\|\right)\right)^{2} /\left(2 \sigma^{2}\right)\right)$, since it has been proven a good choice for hyperspectral images [9]. Two parameters need to be tuned in our kernel versions: the regularization parameter $\lambda$ and the kernel parameter. In this case we used $\lambda=10^{-5} / n$ where $n$ is the number of training samples, and used a isotropic kernel function, whose lengthscale $\sigma$ parameter is tuned in the range of 0.05-0.95 percentiles of the distances between all training samples. We should note that, when a linear kernel is used, $k\left(\mathbf{x}_{i}, \mathbf{x}_{j}\right)=\mathbf{x}_{i}^{\top} \mathbf{x}_{j}$, the proposed algorithms reduce to the linear counterparts proposed in [57]. The SAM kernel approximates the linear kernel for high $\sigma$ values, therefore results should be improved with regard the linear versions. Working in the dual (or $Q$-mode) with the linear kernel instead of the original linear versions can be advantageous only in the case of higher dimensionality than available samples, $d \geq n$.

\footnotetext{
${ }^{4}$ http://isp.uv.es/kacd.html.
} 

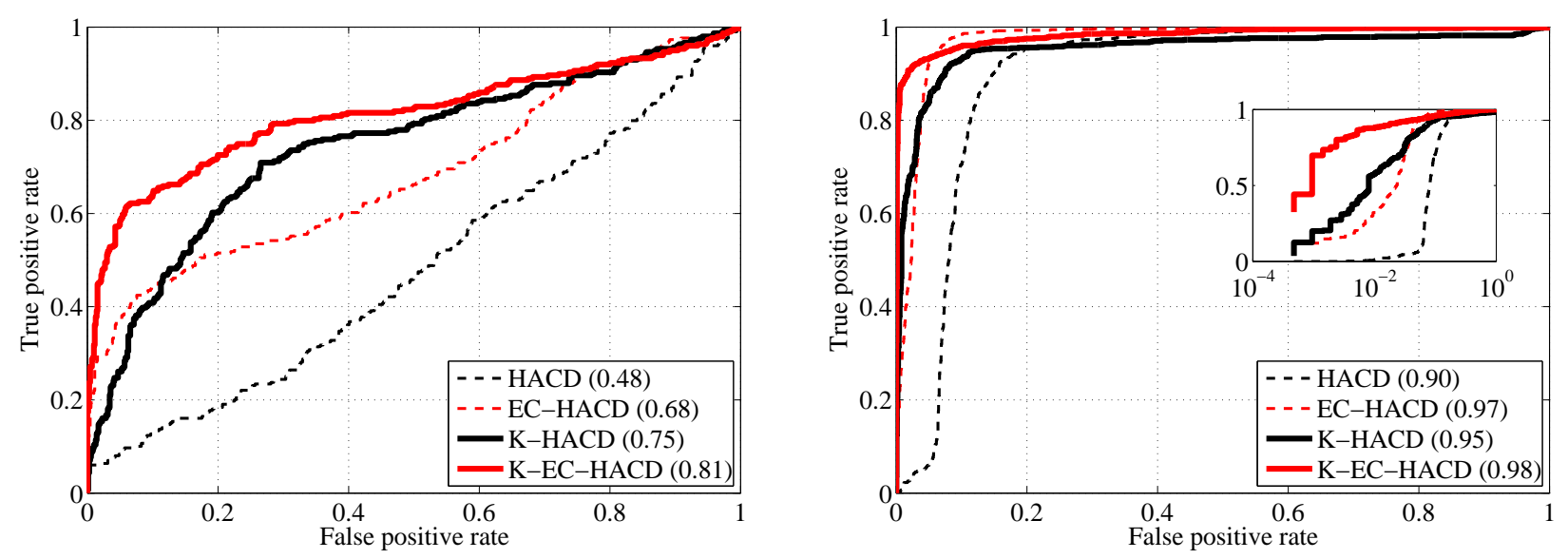

Figure 4: ROC curves and AUC obtained for simulated changes on hyperspectral image. Results using the HACD detector in his linear (Gaussian and EC) and kernelized version are given. Left: results for 100 training examples. Right: results 500 training examples, a version of the plot in logarithmic scale is shown in the detailed plot.

Figure 4 shows the obtained ROC curves for the linear and kernel HACD methods. The dataset was split into small training sets of only 100 and 500 pixels, and results are given for 3000 test samples. The main conclusions are that 1) the kernel versions improve upon their linear counterparts (between 13-26\% in Gaussian and 1-5\% in EC detectors); 2) the EC variants outperform their Gaussian counterparts, especially in the low-sized training sets $(+30 \%$ over HACD and $+18 \%$ over EC-HACD in AUC terms); and 3) results improve for all methods when using 500 training samples. The EC-HACD is very competitive compared to the kernel versions in terms of AUC, but still the proposed K-ECHACD leads to longer tails of false positive detection rates (right figure, inset plot in log-scale).

\subsection{Experiment 2: Real and enforced Changes}

This experiment is designed to analyze the performance of the proposed methods on distortions that are present in real world imagery. While the distortions that are present in any given pair of image sets are location and sensor dependant, some of the more prevalent distortions are due to seasonality, look-angle, and spatial resolution. These experiments employee a very-high spatial resolution sensor that was used to image the same target with highly varying view angles (thus, varying distortion and layover) as well as large differences in seasonality. The ability to detect anomalous changes in these highly distorted image sets illustrates the unique advantage of these types of algorithms and, in particular, the performance advantages of the proposed methods.

The experiments utilize three WorldView-2 images collected in May, August, and November of 2013. All three images (Fig. 5) were collected over a mixed suburban and rural area with urban residential features, roadways, rivers, and agricultural fields. The first image (May) was acquired at a relatively small off-nadir $\left(14.0^{\circ}\right)$ angle early in the summer season. The second (Aug) and third (Nov) images were collected at much higher off nadir angles, $43.6^{\circ}$ and $29.3^{\circ}$, respectively. In each of the final two images, one dark and one white tarp $(20 \times 20 \mathrm{~m}$ each $)$ were introduced as anomalous changes.

This creates two anomalous change image sets on which to test the proposed methods with varying degree of both angular and seasonality distortions: (1) May/Aug: High off-nadir difference, moderate seasonality change; and (2) May/Nov: Moderate off-nadir difference, large seasonality change. While the white and black tarps that are introduced into the change images are highly anomalous, the spectral change is not unrepresentative of real-world problems. Additionally, the ability to more accurately model changes in highly distorted images provides a unique test case for these proposed methods.

For each experiment, 50 non-anomalous pixels were randomly selected from the stacked image sets to model the data space using the proposed algorithms. 500 randomly selected (training samples held out) non-anomalous and all anomalous pixels (May/Aug:153, May/Nov:144) were select for testing. These random selections were collected for 50 independent runs. The mean ROC curves are reported in Fig. 6 and the statistics for AUC are reported in Table 2. As was reported earlier, the parameters $\nu$ and $\sigma$ were tuned through standard cross-validation. The results are shown for independent test sets. In both of the experiments, the HACD and EC-HACD methods had almost identical average ROC curves. The parameter search for $\nu$ used in the EC-HACD method favored very large values, indicating that 

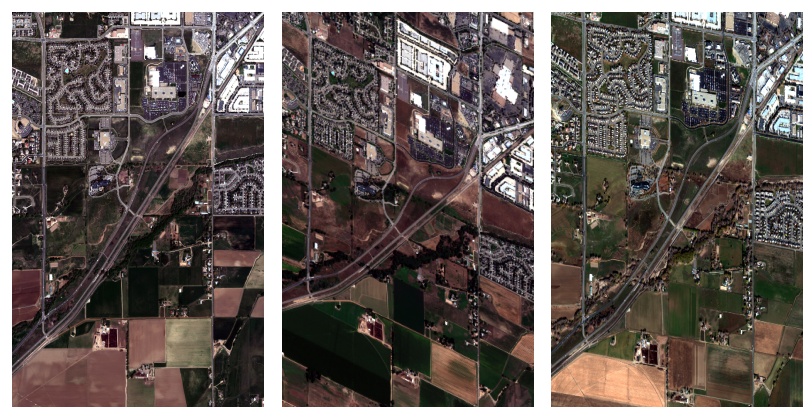

$\begin{array}{lll}\text { (a) May. 2013, (b) Aug. 2013, } & \text { (c) Nov. 2013, } \\ 14.0^{\circ} \text { off-nadir } & 43.6^{\circ} \text { off-nadir } & 29.3^{\circ} \text { off-nadir }\end{array}$

Figure 5: The three WorldView-2 images present a wide variety of distortions due to both seasonality and view angle. In addition to the more obvious changes in agricultural and natural vegetation, the varying view-angles result in variations in ground sample distances (GSD) of $2.0 \mathrm{~m}$ (May), $3.6 \mathrm{~m}$ (Aug), and $2.4 \mathrm{~m}$ (Nov).

Table 2: Area Under the Curve Statistics for the WorldView-2 View-Angle and Seasonality Experiments.

\begin{tabular}{|l|c|c|}
\hline \hline \multicolumn{1}{|c|}{ METHODS } & May-Aug Large Off-Nadir & May-Nov Large Seasonality \\
\hline \hline \multicolumn{3}{|c|}{ Longmont, Colorado } \\
\hline \hline HACD & $0.90 \pm 0.06$ & $0.77 \pm 0.08$ \\
\hline EC-HACD & $0.91 \pm 0.06$ & $0.78 \pm 0.08$ \\
\hline K-HACD & $0.97 \pm 0.04$ & $0.83 \pm 0.11$ \\
\hline K-EC-HACD & $0.99 \pm 0.02$ & $0.95 \pm 0.04$ \\
\hline
\end{tabular}

Table 3: Images attributes in the experimentation dataset.

\begin{tabular}{|c|c|c|c|c|}
\hline Images & Sensor & Size & Bands & SR \\
\hline \multicolumn{5}{|l|}{ Experiment 1 } \\
\hline$\overline{\mathrm{KSC}}$ & AVIRIS & $512 \times 614$ & 224 & $18 \mathrm{~m}$ \\
\hline \multicolumn{5}{|l|}{ Experiment 2} \\
\hline 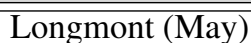 & Worldview-2 & $1156 \times 1563$ & $\overline{88}$ & $2.0 \mathrm{~m}$ \\
\hline Longmont (Aug) & Worldview-2 & $710 \times 1021$ & 8 & $3.6 \mathrm{~m}$ \\
\hline Longmont (Nov) & Worldview-2 & $1074 \times 1149$ & 8 & $2.4 \mathrm{~m}$ \\
\hline \multicolumn{5}{|l|}{ Experiment 3} \\
\hline Argentina & Sentinel-2 & $1257 \times 964$ & 12 & 10m-60m \\
\hline Australia & Sentinel-2 & $1175 \times 2031$ & 12 & $10 \mathrm{~m}-60 \mathrm{~m}$ \\
\hline California & Sentinel-2 & $332 \times 964$ & 12 & $10 \mathrm{~m}-60 \mathrm{~m}$ \\
\hline Poopo Lake & MODIS $^{5}$ & $326 \times 201$ & 7 & $250 \mathrm{~m}-1 \mathrm{~km}$ \\
\hline Denver & QuickBird & $500 \times 684$ & 4 & $1 \mathrm{~m}-4 \mathrm{~m}$ \\
\hline
\end{tabular}

the data space is Gaussian and does not particularly benefit from elliptical modeling. This is most likely due to the anomalousness of the tested anomalous targets. Each of the tarp spectral signatures are highly anomalous (very dark and very bright) presenting a relatively simplified modeling space. However, the kernel methods did outperform the non-kernel methods by a statistically significant $+8 \%$ and $+17 \%$ as measured by mean AUC.

\subsection{Experiment 3: Real and Natural Changes}

This experiment deals with the detection of anomalous changes that can be found naturally in a real environment.

\subsubsection{Data collection}

We collected pairs of multispectral images, each pair consists of images taken at the same location but at different times. We selected the images in such a way that an anomalous change happened between the two acquisition times.

\footnotetext{
${ }^{5}$ Only bands in the visible part of the spectrum were used.
} 
A PREPRINT - DECEMBER 10, 2020

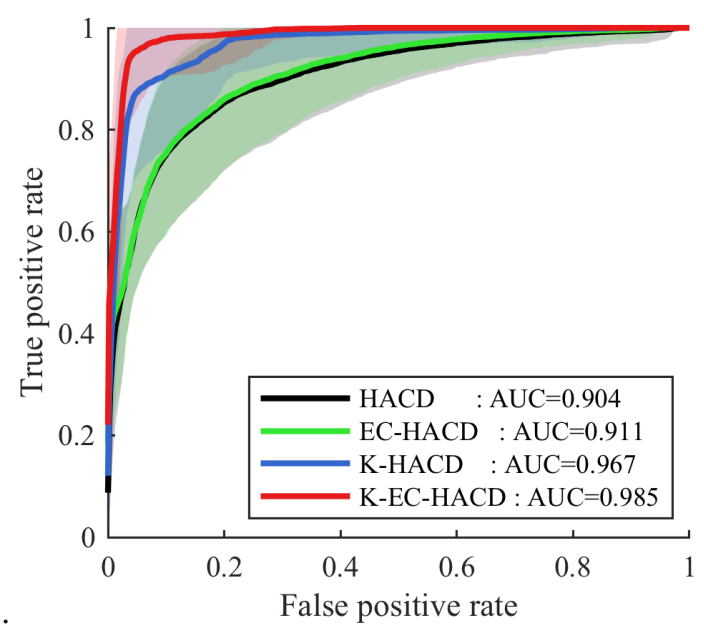

(a) May/Aug High Off-Nadir Experiment

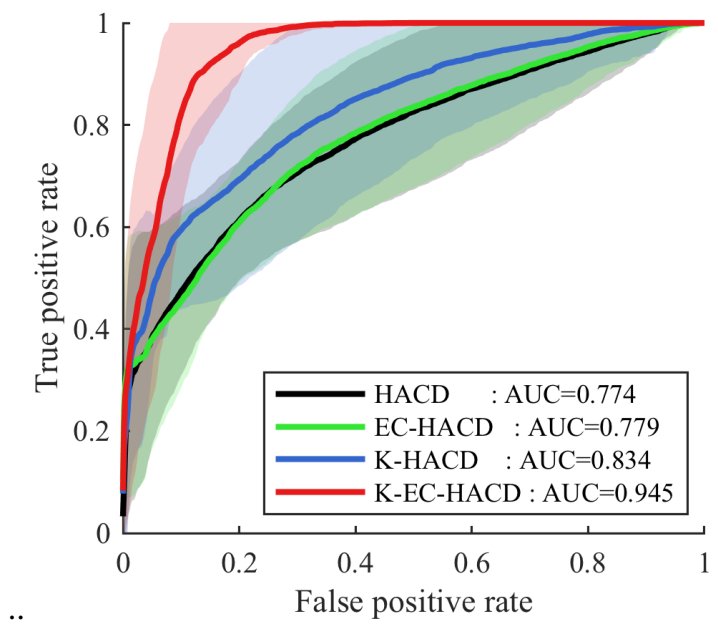

(b) May/Nov Large Seasonality Experiment

Figure 6: ROC curves for the two experiments of Section 3.2. The mean value of the experimental runs is plotted with the standard deviation of each detection algorithm represented by the shaded region.

We manually labeled the ground truth of each pair of images by photo-interpretation, and consequently we know where the anomalous change is present. All images contain changes of different nature, which allow us to study how the different algorithms perform in a diversity of realistic scenarios. Table 3 exposes different descriptors of the images in the database. Fig. 7 shows the RGB composites of the pairs of images and the corresponding groundtruth.

\subsubsection{Numerical comparison}

Different considerations have to be taken when using the different algorithms. On the one hand the family of methods based on EC distribution involve the optimization of the $\nu$ parameter. On the other hand kernel methods involve fitting the kernel function parameters. In this experiment we use the classical $r b f$ kernel which is well suited for multispectral images and has only one parameter, $\sigma$. In addition an extra parameter $\lambda$ has to be fitted to regularize the matrix inversion. Selecting properly all these three parameters is an issue. An ideal situation would be having a rule of thumb to choose them. We performed preliminary experiments to explore the applicability of several existing rules to estimate the $\sigma$ parameter. For the different images and problems faced in this section we applied the heuristics and tried to find an heuristic for the $\nu$ and $\lambda$ parameters. In particular we investigated ten different heuristics: average distance between all samples, median of the distance between all samples, squared root of the dimensionality times variance per dimension averaged, median of the Silverman's rule [51], median of the Scott's rule per feature [48], 
Table 4: AUC results for all five images. First and second best values for each image and each member of the family are in bold. We provide the mean and the standard deviation (in parenthesis) for ten different trials, values marked with (*) had an outlier so we give the median instead of the mean.

\begin{tabular}{|l|l|l|l|l|}
\hline \hline METHODS & RX & YX & XY & HACD \\
\hline \hline \multicolumn{5}{|c|}{ ARGENTINA } \\
\hline ACD & $0.88 \pm 0.008$ & $0.86 \pm 0.010$ & $0.95 \pm 0.004$ & $\mathbf{0 . 9 3} \pm 0.007$ \\
\hline K-ACD & $\mathbf{0 . 9 3} \pm 0.009$ & $\mathbf{0 . 9 4} \pm 0.007$ & $\mathbf{0 . 9 5} \pm 0.011$ & $\mathbf{0 . 9 3} \pm 0.005$ \\
\hline EC-ACD & $0.88 \pm 0.008$ & $0.86 \pm 0.010$ & $0.95 \pm 0.004$ & $0.93 \pm 0.006$ \\
\hline K-EC-ACD & $\mathbf{0 . 9 3} \pm 0.009$ & $\mathbf{0 . 9 4} \pm 0.008$ & $\mathbf{0 . 9 6} \pm 0.008$ & $\mathbf{0 . 9 5} \pm 0.007$ \\
\hline \hline \multicolumn{5}{|c|}{ AUSTRALIA } \\
\hline ACD & $0.79 \pm 0.019$ & $0.79 \pm 0.018$ & $0.83 \pm 0.015$ & $0.79 \pm 0.012$ \\
\hline K-ACD & $\mathbf{0 . 9 2} \pm 0.010$ & $\mathbf{0 . 8 2} \pm 0.019$ & $\mathbf{0 . 8 3} \pm 0.049$ & $\mathbf{0 . 8 9} \pm 0.010$ \\
\hline EC-ACD & $0.79 \pm 0.019$ & $0.80 \pm 0.018$ & $0.83 \pm 0.015$ & $0.80 \pm 0.012$ \\
\hline K-EC-ACD & $\mathbf{0 . 9 2} \pm 0.010$ & $\mathbf{0 . 8 6} \pm 0.016$ & $\mathbf{0 . 9 5} \pm 0.008$ & $\mathbf{0 . 8 7} \pm 0.038$ \\
\hline \hline \multicolumn{5}{|c|}{ CALIFORNIA } \\
\hline ACD & $0.50 \pm 0.015$ & $0.59 \pm 0.017$ & $0.65 \pm 0.018$ & $0.81 \pm 0.014$ \\
\hline K-ACD & $\mathbf{0 . 6 1} \pm 0.024$ & $\mathbf{0 . 7 1} \pm 0.048$ & $\mathbf{0 . 8 5} \pm 0.022$ & $\mathbf{0 . 8 4} \pm 0.013$ \\
\hline EC-ACD & $0.50 \pm 0.015$ & $0.59 \pm 0.016$ & $0.66 \pm 0.024$ & $0.82 \pm 0.016$ \\
\hline K-EC-ACD & $\mathbf{0 . 6 1} \pm 0.024$ & $\mathbf{0 . 7 1} \pm 0.047$ & $\mathbf{0 . 8 5} \pm 0.022$ & $\mathbf{0 . 8 4} \pm 0.013$ \\
\hline \hline \multicolumn{5}{|c|}{ DENVER } \\
\hline ACD & $0.95 \pm 0.013$ & $0.94 \pm 0.014$ & $0.82 \pm 0.059$ & $0.75 \pm 0.058$ \\
\hline K-ACD & $\mathbf{0 . 9 6} \pm 0.023$ & $* \mathbf{0 . 9 4} \pm 0.050$ & $\mathbf{0 . 8 7} \pm 0.017$ & $\mathbf{0 . 9 6} \pm 0.017$ \\
\hline EC-ACD & $0.95 \pm 0.013$ & $0.95 \pm 0.011$ & $0.88 \pm 0.027$ & $0.89 \pm 0.023$ \\
\hline K-EC-ACD & $\mathbf{0 . 9 6} \pm 0.019$ & $* \mathbf{0 . 9 5} \pm 0.037$ & $\mathbf{0 . 9 7} \pm 0.018$ & $\mathbf{0 . 9 7} \pm 0.018$ \\
\hline \hline \multicolumn{5}{|c|}{ POOPO LAKE } \\
\hline ACD & $0.99 \pm 0.002$ & $0.98 \pm 0.003$ & $0.96 \pm 0.007$ & $0.63 \pm 0.032$ \\
\hline K-ACD & $\mathbf{0 . 9 9} \pm 0.002$ & $* \mathbf{0 . 9 7} \pm 0.044$ & $\mathbf{0 . 9 6} \pm 0.007$ & $\mathbf{0 . 9 6} \pm 0.005$ \\
\hline EC-ACD & $0.99 \pm 0.002$ & $0.98 \pm 0.004$ & $0.97 \pm 0.006$ & $0.79 \pm 0.034$ \\
\hline K-EC-ACD & $\mathbf{0 . 9 9} \pm 0.002$ & $\mathbf{0 . 9 8} \pm 0.013$ & $\mathbf{0 . 9 9} \pm 0.002$ & $\mathbf{0 . 9 8} \pm 0.004$ \\
\hline
\end{tabular}

maximum likelihood density estimation, maximum Bayes estimate, maximum entropy estimate, average estimate of marginal kernel density estimate, and kernel density estimation using Gaussian kernel. While some of them have good performance for particular problems none of the rules was useful in general (results not shown). This is a usual problem in ACD where for instance, instead of setting a particular anomaly threshold, it is usual to compute the ROC curve where all the thresholds are evaluated [57]. Since we found that all the rules failed we proposed to use a crossvalidation scheme to fit all the involved parameters: $\sigma, \lambda$, and $\nu$. The same procedure was used for all the algorithms. For each pair of images, we split them into two parts, and we use one for training and one for testing. We select the best parameters by greed search in a cross-validation scheme, using 1000 training samples and 4000 validation samples randomly selected form the training set. Each method implies different set of parameters. For the $\nu$ parameter we explore 100 points logarithmically spaced between $\left[10^{-5}, 10^{10}\right]$. For $\sigma$ parameter, we explore around the heuristic of the mean of the Euclidean distance between pairs of points (which was the most successful in the preliminary experiments), we make a grid by taking 60 logarithmically spaced points respectively between $\left[10^{-3}, 10^{3}\right]$ multiplied by the heuristic value. For the $\lambda$ parameter we use 30 values logarithmically spaced between $\left[10^{-10}, 10^{2.5}\right]$. We optimized the different methods by maximizing the area under the curve (AUC) of the receiver operating characteristic (ROC) curve and use the best parameters in the training set for the validation set.

In Fig. 8 the ROC curves for all the images and all the families of methods are shown. Table 4 summarizes the AUC values of these ROC curves. Fig. 9 shows an example of the spatial distribution of the predictions for the HACD family. We use three different thresholds: one that produces a high rate of detection, one which detect the exact proportion of anomalous changes present in the groundtruth (82\%), and finally one that produces low detection rate. It can be seen how even in low and high detection rates the kernel methods produce more spatially compact detections which brings to much more realistic maps. Fig. 9 compares the ability of the best linear method against the best kernel method when using the optimal threshold. Again kernel methods produce maps with less both false positives and negative alarms.

As a summary, the kernel version achieves the best results in all the images when compared with its linear counterpart. Of the 16 detectors under study there is not an overall winner because each detector has its own characteristics (that can relatively fit data particularities), and the parameters are adjusted according to the type of image. We can see that the K-ACD version obtains better performance both over the linear ACD, and over the linear EC-ACD. And the K-EC-ACD versions have better performance than the rest. For each type of detector (i.e. RX, XY, YX, or HACD) the AUC values can be ranked as: $\mathrm{K}-\mathrm{EC}-\mathrm{ACD} \geqslant \mathrm{K}-\mathrm{ACD} \geqslant \mathrm{EC}-\mathrm{ACD} \geqslant \mathrm{ACD}$. 


\section{Conclusions}

We introduced a family of kernel-based anomaly change detection algorithms. The family extends standard methods like the RX detector [29,44], and many others in the literature [56,57]. The key in the proposed methodology is to redefine the anomaly detection in a reproducing kernel Hilbert space where the data are mapped to. This endorses the methods with improved capacity and flexibility since nonlinear feature relations (and hence anomalies) can be identified. The introduced methods generalize the previous ones since they account for higher-order dependencies between features. We provided implementations of the methods and a database of pairs of images with anomalous changes that can be found in real scenarios ${ }^{6}$.

In practical terms, kernel ACD methods presented here yielded improved results over their linear counterparts in multiand hyperspectral images in both real and simulated, pervasive and anomalous, changes. Results in a wide range of problems with varying complexity (control or not over the anomaly), dimensionality (multi- and hyperspectral sensors), as well as exposure to real and/or pervasive anomalies were presented. We adopted standard metrics for measure and compare methods robustness (AUC and detection) and averaged results over several runs to avoid skewed conclusions.

Interestingly, the EC assumption may be still valid in Hilbert spaces, especially when high pervasive distortions mask anomalous targets. This observation opens the door to the study of the anomalies distribution in Hilbert spaces in the future. A second important conclusion of this work to be highlighted is that, among all 16 methods implemented, we did not observe an overall winner in all situations. After all, each problem has its own characteristics and the different methods adapt to different particularities. In the future we plan to extend the study with low-rank, sparse and scalable kernel versions to cope with high computational requirements.

\section{Acknowledgements}

Research funded by the European Research Council (ERC) under the ERC-CoG-2014 SEDAL project (grant agreement 647423) and the Spainish Ministry of Economy, Industry and Competitiveness under the 'Network of Excellence' program (grant code TEC2016-81900-REDT). Jose A. Padrón was supported by the Grisolia grant from Generalitat Valenciana (GVA) with code GRISOLIA/2016/100.

\section{References}

[1] M. A. Aizerman, E. M. Braverman, and L.I. Rozoner. Theoretical foundations of the potential function method in pattern recognition learning. Automation and remote Control, 25:821-837, 1964.

[2] J. Arenas-García, K. Brandt Petersen, G. Camps-Valls, and L. Kai Hansen. Kernel multivariate analysis framework for supervised subspace learning. IEEE Sig. Proc. Mag., 30(4), 2013.

[3] S. G. Beaven, D. Steind, and L. E. Hoff. Comparison of Gaussian mixture and linear mixture models for classification of hyperspectral data. In IEEE Geosc. Rem. Sens. Symp. (IGARSS), 2000.

[4] D. W. Behnken and N. R. Draper. Residuals and their variance patterns. Technometrics, 14:102 - $111,1972$.

[5] F. Bovolo. A multilevel parcel-based approach to change detection in very high resolution multitemporal images. IEEE Geosci. Remote Sens. Lett., 6(1):33-37, 2009.

[6] F. Bovolo and L. Bruzzone. A split-based approach to unsupervised change detection in large size multitemporal images: application to Tsunami-damage assessment. IEEE Trans. Geosci. Rem. Sens., 45(6):1658-1671, 2007.

[7] F. Bovolo, L. Bruzzone, and S. Marchesi. Analysis and adaptive estimation of the registration noise distribution in multitemporal VHR images. IEEE Trans. Geosci. Rem. Sens., 47(8):2658-2671, 2009.

[8] S. Cambanis, S. Huang, and G. Simons. On the theory of elliptically contoured distributions. J. Multiv. Anal., 11(3):368-385, September 1981.

[9] G. Camps-Valls. Kernel spectral angle mapper. IEE Electronics Letters, 52(14):1218-1220, June 2016.

[10] G. Camps-Valls and L. Bruzzone. Kernel Methods for Remote Sensing Data Analysis. John Wiley and Sons, 2009.

[11] M. J. Canty and A. A. Nielsen. Automatic radiometric normalization of multitemporal satellite imagery with the iteratively re-weighted MAD transformation. Remote Sens. Environ., 112(3):1025-1036, 2008.

\footnotetext{
${ }^{6}$ http://isp.uv.es/kacd.html
} 
[12] M.J. Carlotto. A cluster-based approach for detecting man-made objects and changes in imagery. IEEE Trans. Geosci. Rem. Sens., 43(2):374-387, Feb 2005.

[13] T. Celik. Multiscale change detection in multitemporal satellite images. IEEE Geosci. Remote Sens. Lett., 6(4):820-824, Oct 2009.

[14] C.I. Chang and S.S. Chiang. Anomaly detection and classification for hyperspectral imagery. IEEE Trans. Geosci. Rem. Sens., 40(6):1314-1325, 2002.

[15] J. Chen, X. Chen, X. Cui, and J. Chen. Change vector analysis in posterior probability space: A new method for land cover change detection. IEEE Geosci. Remote Sens. Lett., 8(2):317-321, 2010.

[16] R. Dennis Cook. Detection of influential observation in linear regression. Technometrics, 19(1):15 - 18, 1977.

[17] P. Coppin, I. Jonckheere, K. Nackaerts, and Muys B. Digital change detection methods in ecosystem monitoring: A review. Int. J. Remote Sens, 25(9):1565-1596, 2004.

[18] M. Dalla Mura, J. A. Benediktsson, F. Bovolo, and L. Bruzzone. An unsupervised technique based on morphological filters for change detection in very high resolution images. IEEE Trans. Geosci. Rem. Sens., 5(3):433-437, 2008.

[19] M. Ding, Z. Tian, Z. Jin, M. Xu, and C. Cao. Registration using robust kernel principal component for objectbased change detection. IEEE Geoscience and Remote Sensing Letters, 7(4):761-765, Oct 2010.

[20] S. Ghosh, L. Bruzzone, S. Patra, F. Bovolo, and A. Ghosh. A context-sensitive technique for unsupervised change detection based on Hopfield-type neural networks. IEEE Trans. Geosci. Rem. Sens., 45(3):778-789, 2007.

[21] L. Gómez-Chova, A.A. Nielsen, and G. Camps-Valls. Explicit signal to noise ratio in reproducing kernel Hilbert spaces. In IEEE Geosc. Rem. Sens. Symp. (IGARSS), pages 3570-3570. IEEE, Jul 2011.

[22] A. A. Green, M. Berman, P. Switzer, and M. D. Craig. A transformation for ordering multispectral data in terms of image quality with implications for noise removal. IEEE Trans. Geosc. Rem. Sens., 26(1):65-74, 1998.

[23] J. Im, J. R. Jensen, and M. E. Hodgson. Optimizing the binary discriminant function in change detection applications. Remote Sens. Environ., 112(6):2761-2776, 2008.

[24] H. Kwon, S.Z. Der, and N.M. Nasrabadi. Adaptive anomaly detection using subspace separation for hyperspectral images. Opt. Eng., 42(11):3342-3351, 2003.

[25] H. Kwon and N. Nasrabadi. Kernel RX-algorithm: a nonlinear anomaly detector for hyperspectral imagery. IEEE Trans. Geosci. Rem. Sens., 43(2):388-397, 2005.

[26] H. Kwon and N.M. Nasrabadi. Kernel RX-algorithm: a nonlinear anomaly detector for hyperspectral imagery. IEEE Trans. Geosc. Rem. Sens., 43(2):388-397, 2005.

[27] Quin K liu J, Gong M. A deep convolutional coupling network for change detection based on heterogeneous optical and radar images. IEEE Transactions on Neural Networks and Learning Systems., 27:545 - 559, 2018.

[28] D. Lu, P. Mausel, E. Brondízio, and E. Moran. Change detection techniques. Intnl. Jour. Rem. Sens., 25(12):2365-2401, 2004.

[29] X. Lu, L.E. Hoff, I.S. Reed, M. Chen, and L.B. Stotts. Automatic target detection and recognition in multiband imagery: a unified ML detection and estimation approach. IEEE Trans. Image Proc., 6:143-156, 1997.

[30] S Lyu and E P Simoncelli. Nonlinear extraction of 'independent components' of natural images using radial Gaussianization. Neural Computation, 21(6):1485-1519, Jun 2009.

[31] W. A. Malila. Change vector analysis: An approach for detecting forest change with landsat. In IEEE Proceedings of Annual Symposium on Machine Processing of Remotely Sensing Data, pages 326-336, 1980.

[32] D. Manolakis, D. Marden, and Gary A. Shaw. Hyperspectral image processing for automatic target detection applications, 2003.

[33] D. Manolakis and G. Shaw. Detection algorithms for hyperspectral imaging applications. IEEE Signal Processing Magazine, pages 29-43, 2002.

[34] R. Mayer, F. Bucholtz, and D. Scribner. Object detection by using 'whitening/dewhitening' to transform target signatures in multitemporal hyperspectral and multispectral imagery. IEEE Trans. Geosci. Remote Sens., 41(5):1136-1142, May 2003.

[35] A. A. Nielsen. The regularized iteratively reweighted MAD method for change detection in multi- and hyperspectral data. IEEE Trans. Image Process., 16(2):463-478, 2006. 
[36] A. A. Nielsen, K. Conradsen, and J. J. Simpson. Multivariate alteration detection (MAD) and MAF postprocessing in multispectral bi-temporal image data: New approaches to change detection studies. Rem. Sens. Env., 64(1):1-19, April 1998.

[37] A. A. Nielsen, K. Conradsen, and J. J. Simpson. Multivariate alteration detection (MAD) and MAF postprocessing in multispectral, bitemporal image data: New approaches to change detection studies. Remote Sens. Environ., 64:1-19, 1998.

[38] A.A. Nielsen. Kernel maximum autocorrelation factor and minimum noise fraction transformations. IEEE Trans. Image Processing, 20(3):612-624, Mar 2011.

[39] Wang X Ouyang W, Zeng X. Deepid-net: Object detection with deformable part based convolutional neural networks. IEEE Transactions on Pattern Analysis and Machine Intelligence., 39:1320-1334, 2017.

[40] F. Pacifici, M. Chini, C. Bignami, S. Stramondo, and W.J. Emery. Automatic damage detection using pulsecoupled neural networks for the 2009 italian earthquake. In IEEE Geosc. Rem. Sens. Symp. (IGARSS), pages 1996-1999, Jul 2010.

[41] F. Pacifici, F. Del Frate, and W.J. Emery. Pulse coupled neural networks for detecting urban areas changes at very high resolutions. In Urban Remote Sensing Event, 2009 Joint, pages 1-7, May 2009.

[42] R. J. Radke, S. Andra, O. Al-Kofahi, and B. Roysam. Image Change Detection Algorithms: A Systematic Survey. IEEE Trans. Image Process., 14(3):294-307, 2005.

[43] R. J. Radke, S. Andra, O. Al-Kofahi, and B. Roysam. Image change detection algorithms: a systematic survey. IEEE Transactions on image processing, 14(3):294 - 307, 2005.

[44] I. S. Reed and X. Yu. Adaptive multiple-band CFAR detection of an optical pattern with unknown spectral distribution. IEEE Trans. Acoustics, Speech and Signal Process., 38(10):1760-1770, 1990.

[45] I.S. Reed and X. Yu. Adaptive multiple-band CFAR detection of an optical pattern with unknown spectral distribution. IEEE Trans. Acou. Spee. Sig. Proc., 38(10):1760-1770, 1990.

[46] J.L. Rojo-Álvarez, M. Martínez-Ramón, J. Muñoz Marí, and G. Camps-Valls. Digital Signal Processing with Kernel Methods. Wiley \& Sons, UK, Apr 2017.

[47] A. Schaum and A. Stocker. Long-interval chronochrome target detection. In Proc. Int. Symp. Spectral Sens. Res., 1997.

[48] David Scott. Scott's rule. Wiley Interdisciplinary Reviews: Computational Statistics, 2, 2010.

[49] C. E. Shannon. A mathematical theory of communication. Bell Systems Technical Journal, 27:623-656, 1948.

[50] J. Shawe-Taylor and N. Cristianini. Kernel Methods for Pattern Analysis. Cambridge University Press, New York, NY, USA, 2004.

[51] B. W. Silverman. Density Estimation for Statistics and Data Analysis. Chapman \& Hall, London, 1986.

[52] A. Singh. Digital change detection techniques using remotely-sensed data. Int. J. Rem. Sens., 10(6):989-1003, 1989.

[53] J. Theiler. Quantitative comparison of quadratic covariance-based anomalous change detectors. Appl. Opt., 47:F12-F26, 2008.

[54] J. Theiler. Quantitative comparison of quadratic covariance-based anomalous change detectors. Applied Optics, 47(28):12-26, October 2008.

[55] J. Theiler and S. M. Adler-Golden. Detection of ephemeral changes in sequences of images. In 2008 37th IEEE Applied Imagery Pattern Recognition Workshop, pages 1-8, Oct 2008.

[56] J. Theiler and S. Perkins. Proposed framework for anomalous change detection. In Proc. ICML Workshop Mach. Learn. Algorithms Surveillance Event Detection, pages 7-14, 2006.

[57] J. Theiler, C. Scovel, B. Wohlberg, and B. R. Foy. Elliptically contoured distributions for anomalous change detection in hyperspectral imagery. IEEE Geosc. Rem. Sens. Lett., 7(2):271-275, April 2010.

[58] J. Theiler and B. Wohlberg. Local coregistration adjustment for anomalous change detection. IEEE Transactions on Geoscience and Remote Sensing, 50(8):3107-3116, Aug 2012.

[59] M. Volpi, D. Tuia, G. Camps-Valls, and M. Kanevski. Unsupervised change detection with kernels. IEEE Geoscience and Remote Sensing Letters, 9(6):1026-1030, Nov 2012.

[60] M. Volpi, D. Tuia, M. Kanevski, and G. Camps-Valls. Unsupervised change detection by kernel clustering. In Proceedings of the SPIE Remote Sensing Conference, 2010. 
[61] C. Wu, L. Zhang, and B. Du. Kernel slow feature analysis for scene change detection. IEEE Transactions on Geoscience and Remote Sensing, 55(4):2367-2384, April 2017.

[62] Zhang $\mathrm{Z}$ Zhang $\mathrm{C}$. Improving multiview face detection with multi-task deep convolutional neural networks. Applications of Computer Vision (WACV), 2014 IEEE Winter Conference on.

[63] J. Zhou, C. Kwan, B. Ayhan, and M. T. Eismann. A novel cluster kernel rx algorithm for anomaly and change detection using hyperspectral images. IEEE Transactions on Geoscience and Remote Sensing, 54(11):64976504, Nov 2016. 


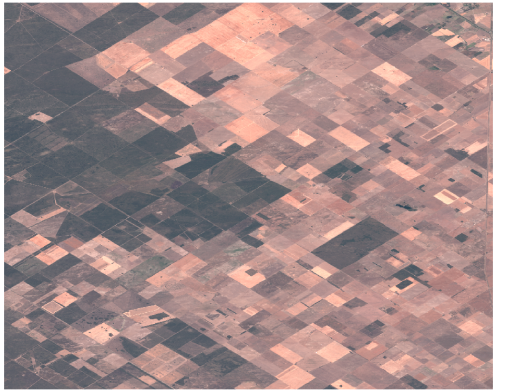

(a) Jul 2016

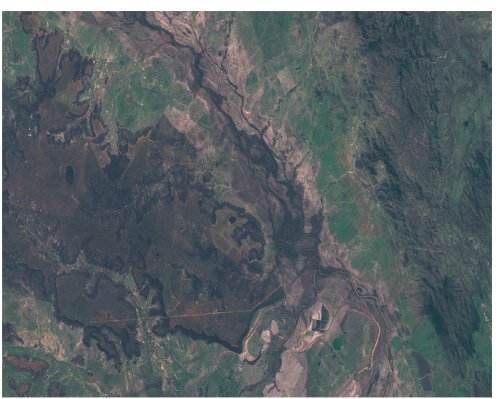

(c) Mar 2017

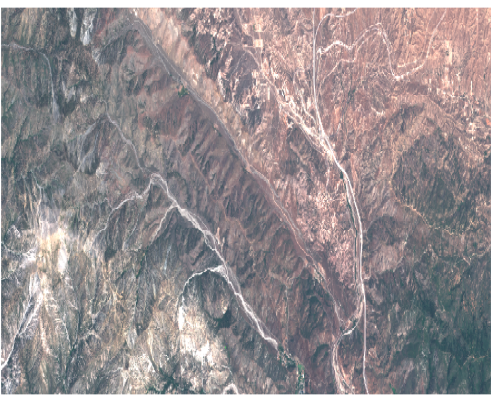

(e) Aug 8th 2017

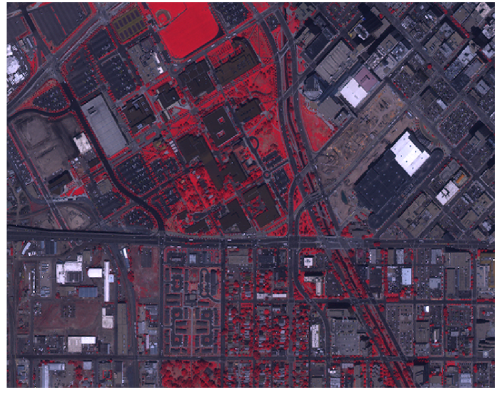

(g) Jul 17th 2002

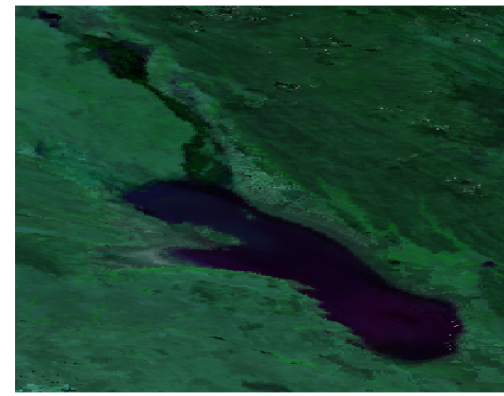

(i) Oct 4th 2015

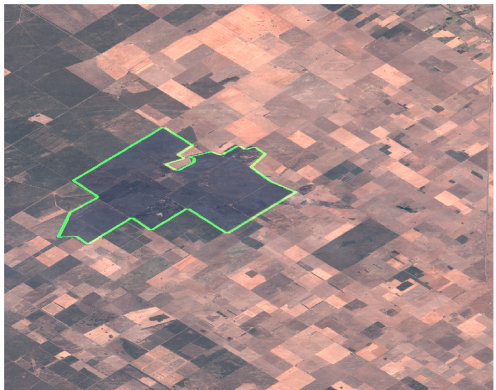

(b) Aug 2016

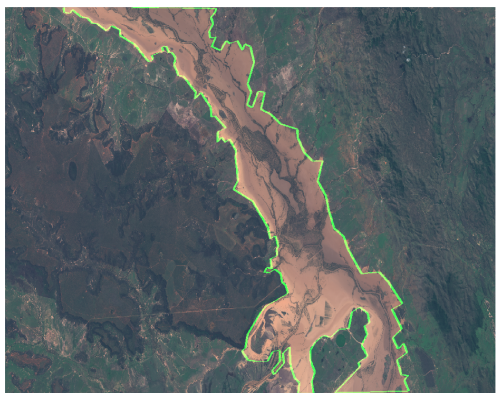

(d) May 2017

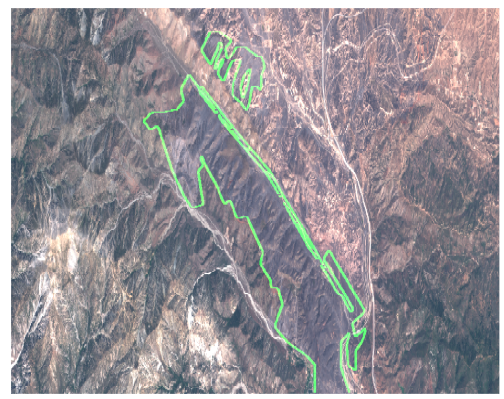

(f) Aug 28th 2017

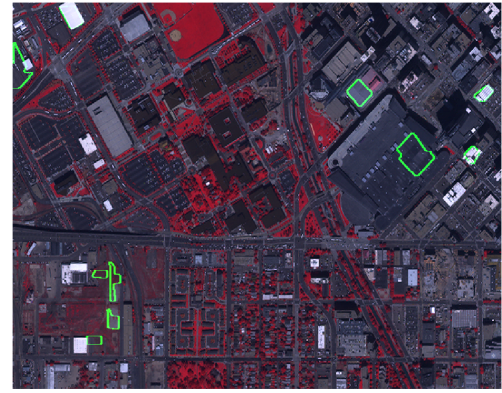

(h) Aug 22nd 2008

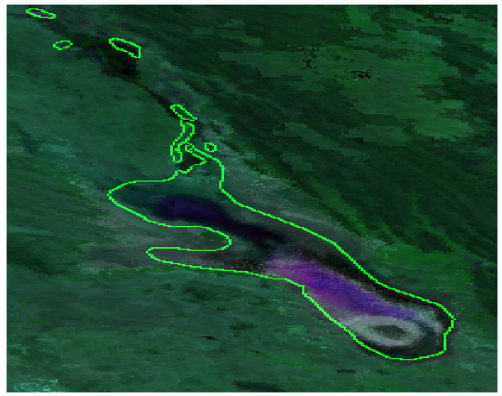

(j) Feb 20th 2016

Figure 7: Images with natural anomalies. Left column: imageq without anomaly. Right column: images with anomalous change and their corresponding ground truth in green. First row: we can see an area burned in Argentina between the months of July and August 2016. Second row: natural floods caused by Cyclone Debbie in Australia 2017. Third row: consequences of the fire in a mountainous area of California (USA). Fourth row: Quickbird multispectral images acquired over Denver city (USA) where appears an urbanized area. Last row: drying of Poopo Lake in Bolivia at the end of 2015. 


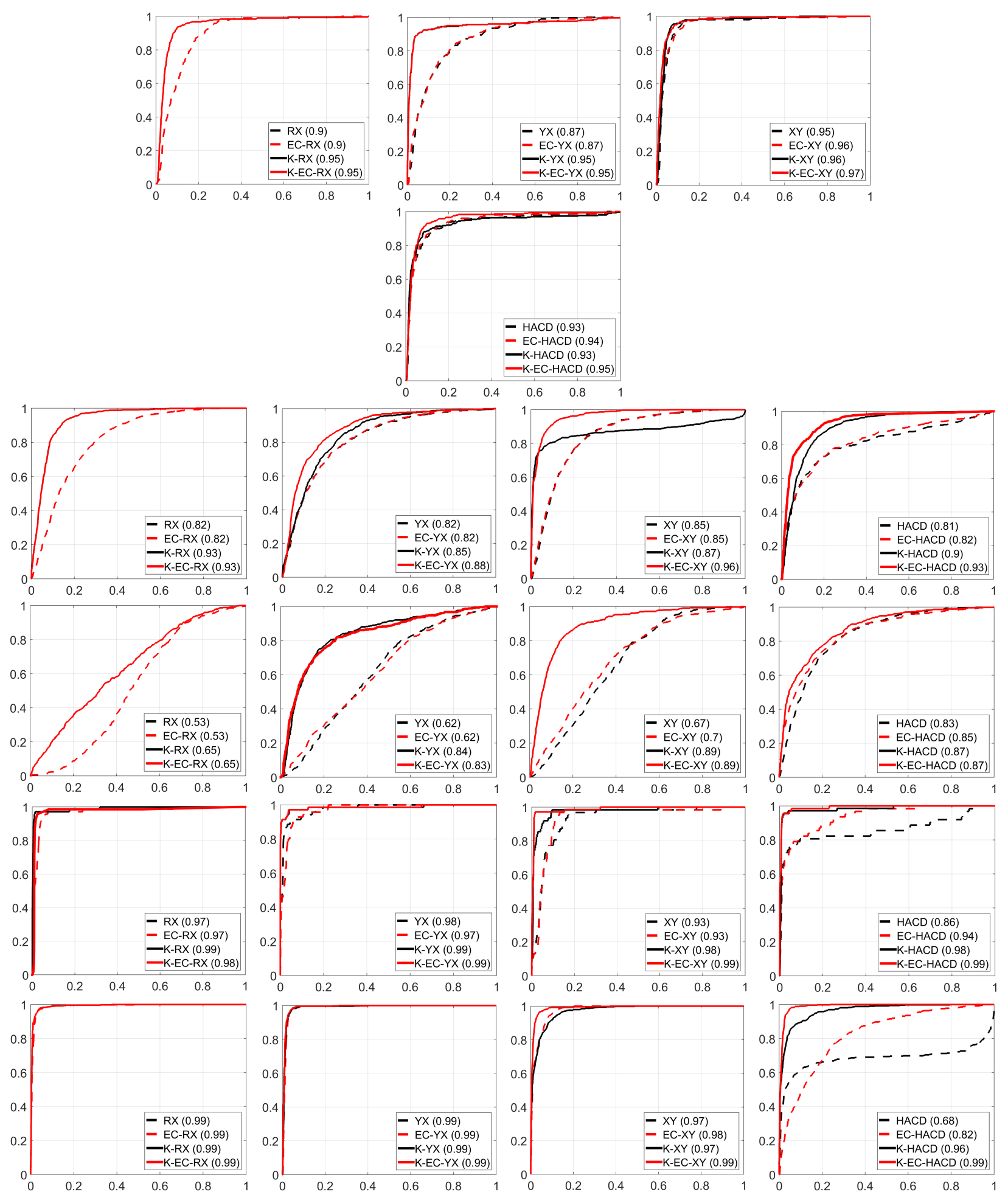

Figure 8: ROC curves and AUC obtained for natural changes on multispectral images. In rows the images from Argentina, Australia, California, Denver to Lake (see table 3 for details) and in the columns from left to right the RX, YX, XY and HACD methods respectively. 


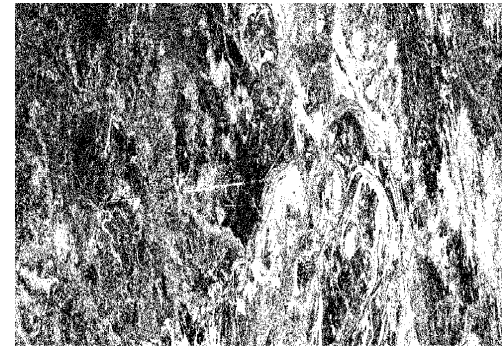

(a) HACD $50 \%$

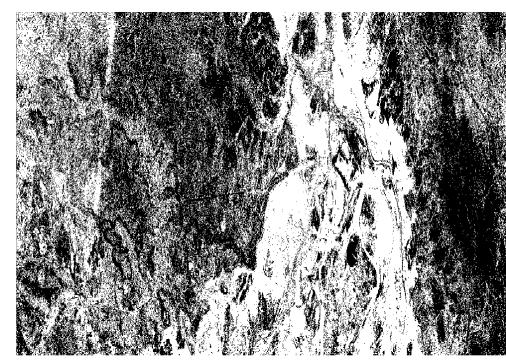

(d) EC-HACD 50\%

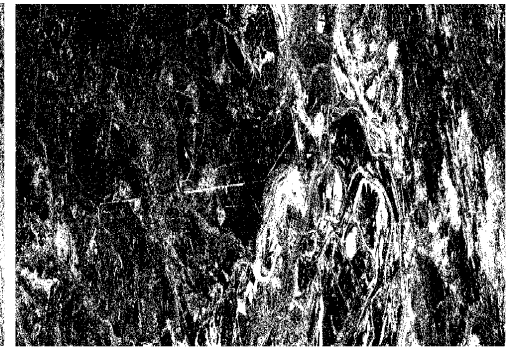

(b) HACD $82 \%$

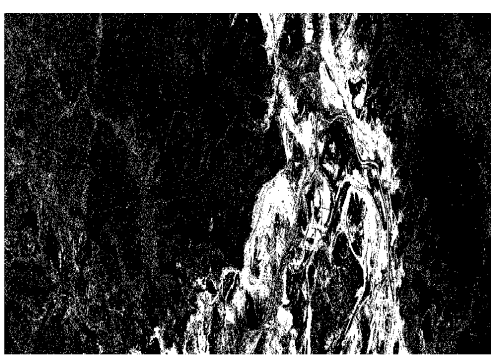

(e) EC-HACD $82 \%$

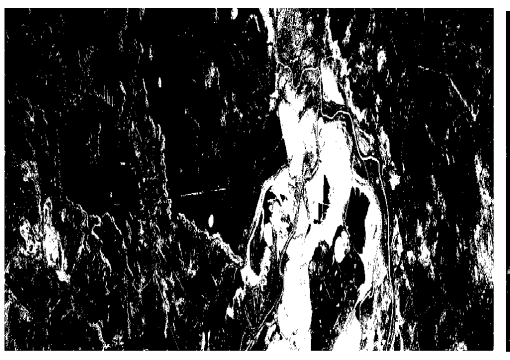

(h) K-HACD $82 \%$

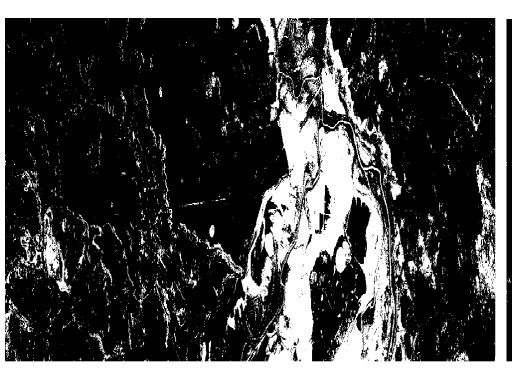

(k) K-EC-HACD $82 \%$

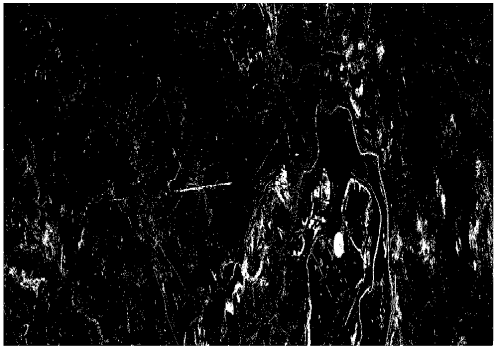

(c) HACD $97 \%$

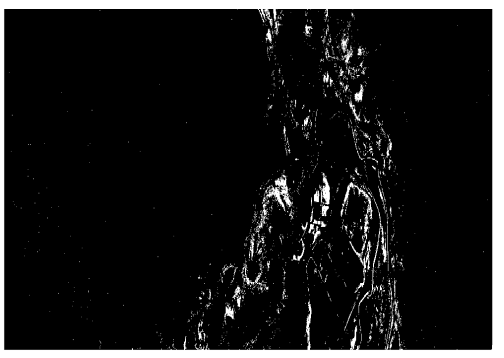

(f) EC-HACD $97 \%$

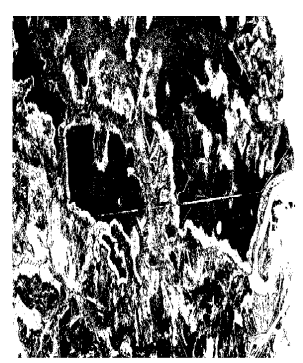

(g) K-HACD $50 \%$

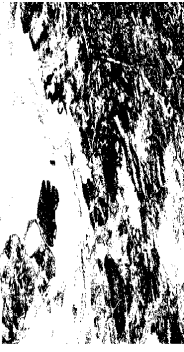

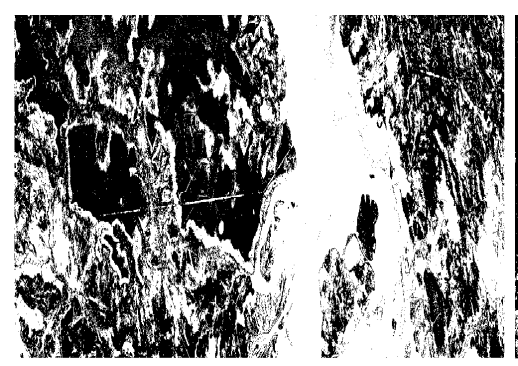

(j) K-EC-HACD $50 \%$

(j)

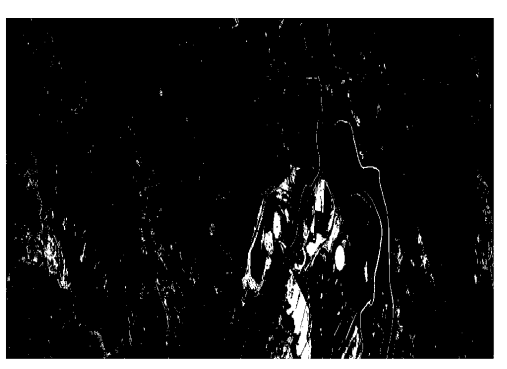

(i) K-HACD $97 \%$

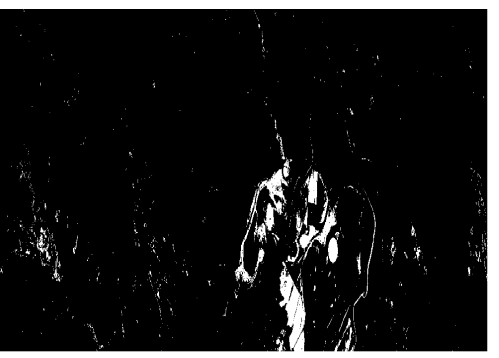

(1) K-EC-HACD $97 \%$

Figure 9: HACD Predictions Maps from Australia test. Left : lower threshold, Center : optimal threshold and Right : high threshold. 

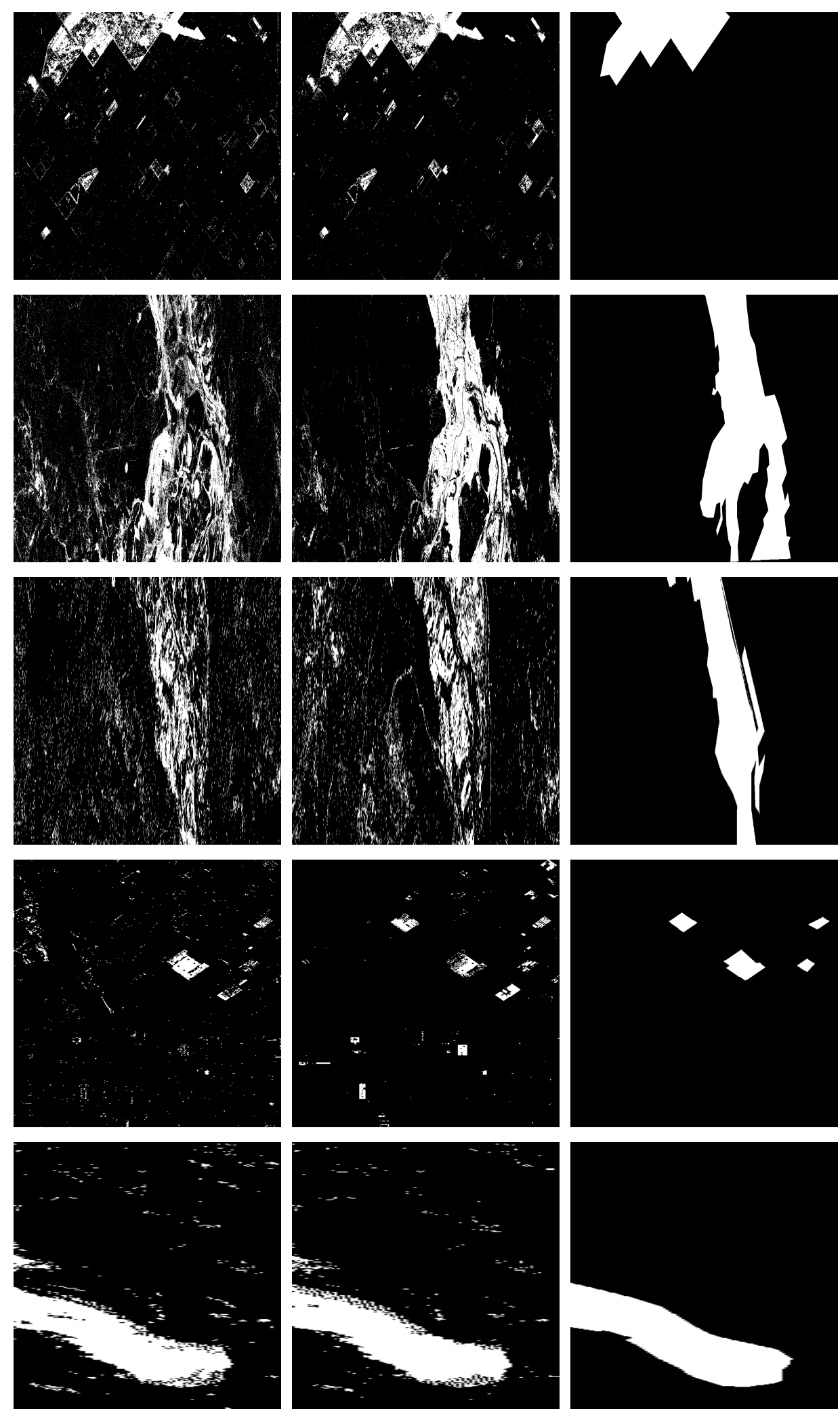

Figure 10: Predictions Maps from best linear and kernel methods. Left column: best linear case, Center column: best kernel case and Right column: ground truth. 